\title{
Psicologia Social de la Salud sobre el consumo adictivo de cocaina: un modelo psicosocial sintético
}

\section{Social Psychology of the Health on the addictive cocaine's consumption: a synthetic psychosocial model}

JESÚS SAIZ GALDÓS*

\section{RESUMEN}

El consumo de drogas es un problema personal y social que se ha abordado desde numerosos frentes y distintas perspectivas. Los estudios sobre los factores de riesgo y protección en el uso y abuso de drogas son abundantes $y$, mientras que en un principio los investigadores anteponían unos factores sobre otros, en estos momentos se está optando cada vez más por una perspectiva integral y sintética.

Este artículo pretende precisamente presentar la perspectiva psicosocial del problema de la adicción a la cocaina, y parte del supuesto de que la Psicología Social posee una capacidad integrativa privilegiada para ofrecer un modelo de análisis lo suficientemente amplio, dentro del cual pueda ocurrir una convergencia de posturas. En primer lugar, se habla de la Teoría de la Conducta Planificada, así como se revisa el Modelo Psicosocial para el Estudio de la Salud Mental. En segundo lugar, se retoma la perspectiva de los estudios de valores y rasgos de personalidad que poseen mayor impacto para el problema de la adicción. Por último, se ofrece un esquema de análisis que integra y resume las aportaciones en el estudio de los factores que afectan al problema de la adicción a la cocaína.

\section{PALABRAS CLAVE}

Cocaína, Psicología Social, Actitudes, Valores, Rasgos de Personalidad.

\footnotetext{
* Psicólogo (M-19131). Departamento de Psicología Social, Facultad de ciencias Políticas y Sociológía, Universidad Complutense de Madrid. Equipo de apoyo Social Comunitario Fuencarral, Consejería de Familia y Asuntos Sociales, Grupo 5, Comunidad de Madrid. jesus_saiz@hotmail.com
} 
Psicología Social de la Salud sobre el consumo adictivo de cocaina: un modelo psicosocial sintético

\section{ABSTRACT}

The drugs consumption is a personal and social problem that has been boarded from numerous fronts and different perspectives. The studies about the risk and protection factors of the use and drug abuse are abundant and, whereas at the beginning the researchers focused in only few factors, at the moment it is being used a more integral and synthetic perspective.

This article indeed tries to present the psychosocial perspective of the problem of cocaine addiction. Here it is assumed that Social Psychology has a privileged integrative capacity, and it can offer an analysis model big enough to make possible a convergence of different positions. In the first place, the Theory of the Planned Behaviour, as well as the Psycho-Social Model for the Study of the Mental Health is reviewed. Secondly, the studies of values and personality traits having greater impact for the problem of drug addiction are also reviewed. Finally, an analysis scheme is offered that integrates and summarizes the contributions in the study of the factors affecting cocaine addiction.

\section{KEY WORDS}

Cocaine, Social Psychology, Attitudes, Values, Personality traits. 


\section{Introducción}

La cocaína es una droga de abuso que produce efectos estimulantes en el sistema nervioso central y su forma más habitual de ser consumida en España es por vía intranasal (clorhidrato de cocaína). Los niveles de preocupación y alerta no pueden ser más elevados, mientras que la Oficina de Naciones Unidas contra las Drogas y el Crimen publicó en su último informe (UNODC, 2007) que somos el país con un mayor porcentaje de consumo de cocaína, la "Encuesta estatal sobre el uso de drogas en enseñanza secundaria" (PND, 2004) reveló que el consumo de cocaina en España ha ido ascendiendo año con año. Además, ha habido un aumento considerable de la población juvenil consumidora $\mathrm{y}$ en el número de personas con problemas por esta sustancia que piden ser atendidos en servicios sanitarios y sociales durante los últimos dos años (OED, 2005).

Tras la preocupación generada por el creciente consumo de cocaína en la sociedad española (Megías, 2004), este artículo tiene por objetivo realizar una puesta al día de aquellas contribuciones teóricas que desde la Psicología Social se pueden aplicar al estudio del consumo de drogas, tanto en su versión preventiva como en intervención directa. ${ }^{1}$

Paradójicamente, a pesar de la consolidada presencia que la Psicología Social posee en el área de la salud y de la salud mental, son pocos los trabajos que se han dedicado al estudio de las drogas. Algunas revisiones generales son las de Strobe y Strobe (1995) o Parrott (1998), mientras que Pons y Berjano (1999) realizaron un estudio psicosocial del consumo de alcohol entre los adolescentes.
Para ocuparnos del problema de las drogas desde una postura psicosocial, será necesario situarnos primeramente dentro de la vertiente de la Psicología Social que se debería ocupar de este tema: la Psicología Social de la Salud y de la Salud Mental. Desde que en 1948, en el Congreso de Constitución de la Organización Mundial de la Salud, se definiese la Salud como: "el estado de absoluto bienestar físico, mental y social, y no meramente la ausencia de enfermedad" la Psicología Social ha tratado de mantener una presencia activa en el campo de la Salud. Según Rodríguez y García (1999, p. 352): "la Psicología Social de la Salud es la aplicación específica de los conocimientos y técnicas de la Psicología Social a la comprensión de los problemas de la salud, y al diseño y puesta en práctica de programas de intervención en ese marco".

Strobe y Strobe (1995) hacen énfasis en la utilidad de los conocimientos psicosociales para el cambio de patrones conductuales no-saludables, la reducción del estrés psicosocial y la promoción de conductas saludables. Mientras que Rutter, Quine y Chesham (1993, p. 55) subrayan: "el rol de los factores psicosociales como mediadores entre las influencias sociales y los resultados para la salud". Aquellos factores que desde la Psicologia Social han recibido más atención son: factores sociales (género y posición social), sucesos vitales estresantes, apoyo social, factores emocionales (depresión, ansiedad, autoestima), conocimiento, información y comunicación (especialmente en la relación médicopaciente), valores, personalidad, disposiciones cognitivas (control personal, creencias y actitudes), estrés, estrategias de afrontamiento (solución de problemas) y conductas apropiadas e inapropiadas

${ }^{1}$ A diferencia de las revisiones de otros autores (Becoña, 1999, 2002), ésta se concentra en las aportaciones de la Psicologia Social. 
para la salud (ejercicio, dieta sana, comportamientos de riesgo, etc.).

Por otra parte, cuando los conocimientos de la Psicología Social se aplican al específico campo de la salud mental, como se detalla más adelante, los resultados que se obtienen pueden ser igualmente satisfactorios (Sánchez, Garrido y Álvaro, 2003).

\section{Modelos Psicosociales aplicados a la Salud y al área de Drogodependencias}

\section{a) La Teoría de la Conducta Planificada}

La Teoría de la Conducta Planificada de Ajzen (1991) es una teoría psicosocial que cuenta con una gran cantidad de apoyo empírico y que ha sido aplicada al área de la salud y de las drogodependencias en repetidas ocasiones. Esta teoria, formulada en principio para predecir las conductas en general, fue elaborada sobre la base de la Teoría de la Acción Razonada (TAR) de Ajzen y Fishbein (1980).

Los tres elementos clave de la TAR son: 1) La Intención, factor más directo y cercano a la conducta. 2) La Actitud, o valoración positiva o negativa que el sujeto hace de la realización de la conducta, la cual a su vez está determinada por las creencias de la persona sobre los resultados del comportamiento y sus evaluaciones de estos resultados. Y 3) la Norma Subjetiva, que consiste en la percepción que el sujeto tiene de las presiones sociales a que realice $u$ omita cierta conducta, y que a su vez está compuesta por las creencias de la persona sobre lo que determinados grupos de referencia piensan de la conducta y su motivación para complacer a estos grupos de referencia. Por último, en un intento por integrar otros factores a su teoria, los autores consideraron que ciertas variables "externas" como características de personalidad, inteligencia, variables sociodemográficas, etc., podrían influir en las creencias de las personas y por este medio en la intención conductual.

Una de las principales limitaciones de esta teoría consistía en que quedaban sin explicación todas aquellas conductas en las que el sujeto no poseía control alguno (como por ejemplo el correr $100 \mathrm{mts}$. en $5 \mathrm{seg}$.$) . Con el fin de$ responder a sus críticos Ajzen (1991) propuso la Teoría de la Conducta Planificada (TCP), la cual sin modificar la anterior sustancialmente, incluye un nuevo elemento al esquema: el Control Conductual Percibido. Éste (Schifter y Ajzen, 1985, p.844): "representa la percepción de la facilidad o dificultad para realizar (una conducta) y se asume que refleja la experiencia pasada así como la anticipación de impedimentos y obstáculos".

Además de las investigaciones de Humphrey, O`Malley, Johnston y Bachman (1988) y Levy y Pierce (1989), un estudio de actualidad, que aplica la TCP al área del consumo de drogas, es el que Hulten et al. (2003) realizaron sobre el uso y abuso de benzodiacepinas (BZP). Los autores exploraron qué factores psicosociales determinan la longitud del periodo del uso de la droga, y para ello utilizaron un cuestionario autoadministrado que aplicaron a 467 sujetos, de entre los cuales 360 tenian experiencia previa con el uso de BZP y 107 no tenian experiencia (pero iban a comenzar a usarla).

La comparación entre las puntuaciones medias de los grupos en el cuestionario, reveló que aquellos que no poseían uso previo de BZP tenian un mayor control conductual, así como estaban más influenciados por la norma social (opi- 
nión de su médico). Mientras que aquellos con experiencia en el uso de BZP tenían mayor intención conductual y actitudes favorables hacia el uso de BZP. Además, los autores pudieron comprobar las siguientes relaciones:

a) En sujetos con experiencia previa, la intención conductual es el elemento que mayor predicción posee sobre el uso de BZP. Las actitudes predicen el uso de BZP por medio de la intención conductual. El control conductual percibido se relaciona directa y negativamente con la conducta, lo cual indicaria que cuando el sujeto piensa que no es capaz de controlar su hábito se abandona al abuso incontrolado. La creencia de que el uso de BZP conlleva adicción determina negativamente el control conductual percibido y positivamente la intención conductual (a menor control, mayor adicción y mayor consumo).

b) Por otro lado, en sujetos sin experiencia previa, las relaciones significativas fueron mucho menores. Entre ellas destacan que la intención determinaba la conducta de consumo, la actitud se relacionaba con la intención de consumo y la norma social determinó la actitud.

Por último, cabe señalarse que aunque el modelo explica el $67 \%$ de la varianza para los consumidores experimentados, sólo explica el 18\% de la varianza para los inexpertos, lo cual sugiere la existencia de otros factores que la teoría no ha tomado en consideración (esta deficiencia ya la han señalado otros autores como Ros, 2001). No obstante, queda demostrado que elementos como la intención conductual, el grado de control percibido, las actitudes y la norma social son muy necesarios para explicar las conductas relacionadas con el uso y abuso de drogas (especialmente cuando el consumo es alto).

\section{b) Modelo Psicosociológico para el Estudio} de la Salud Mental

Existen teorías que definen la salud mental desde una perspectiva psicosocial como el modelo de salud mental de Warr, la teoría de la indefensión de Seligman, el modelo cognitivo de la depresión de Beck o el modelo de la causación social de Mirowsky y Ross (en Álvaro y Páez, 1999). De hecho, de este último se desprende el Modelo Psicosociológico para el Estudio de la Salud Mental propuesto recientemente por Sánchez, Garrido y Álvaro (2003), el cual propone la existencia de una relación lineal entre el nivel estructural (posición social) del sujeto, las condiciones de vida que atraviesa (alineación, estrés), un nivel psicosociológico (estrategias de afrontamiento, autoestima y apoyo social que posee) y la situación o nivel psicológico del individuo (tanto su salud mental como su bienestar psicológico).

Este modelo está basado en una investigación empírica de carácter transversal en la que se aplicó un cuestionario especialmente diseñado a 401 personas españolas. El cuestionario poseía una primera parte que exploraba datos sociodemográficos y una segunda sección que incluía medidas sobre posición social (estatus laboral y nivel de ingresos), salud mental, deterioro psicológico, sucesos vitales estresantes, alineación, autoestima, apoyo social percibido y estrategias de afrontamiento.

Entre los resultados que encontraron los autores, resalta la comprobación de la pertinencia de combinar factores sociodemográficos con factores psicosociales y psicológicos en un modelo de causación social, llegando a concluir que, (Ibíd., p. 28) "a medida que se reduce el nivel socioeconómico, aumentan los niveles de alineación y situaciones estresantes". Sin embargo, esto envuelve asi- 
mismo, una importante limitación en su aplicación al área del consumo de cocaína, y es que, aun pudiendo explicar efectivamente la relación existente entre consumo de cocaina y situación social desfavorecida (desempleo, bajos recursos económicos, etc.), dejaría sin cubrir los motivos de consumo entre un amplio sector proveniente de círculos económicamente privilegiados o de clases económicas altas o medias (que es por otra parte el tipo de consumo que actualmente se está imponiendo).

En resumen, el Modelo Psicosociológico para el Estudio de la Salud Mental propuesto por Sánchez, Garrido y Álvaro (2003), posee la virtud de relacionar variables sociales y psicológicas dentro de un mismo marco conceptual, y recuerda al investigador que en todo momento el sujeto está inmerso en un entorno social o contexto, que va a influir decisivamente sobre su conducta y situación personal.

\section{c) Valores Personales y Drogas}

Dentro de la Psicologia Social, la concepción de los valores personales posiblemente más novedosa, y que cuenta con un mayor apoyo empirico, es la elaborada por Schwartz y Bilsky (1987) y revisada posteriormente por Schwartz (2001). Para este autor, los valores son entendidos como (Schwartz, 2001, p.55): "metas deseables y transituacionales, que varían en importancia, que sirven como principios en la vida de una persona o de otra entidad social".

De esta manera, Schwartz propone la existencia de diez tipos de motivaciones de valor: 1) Poder; estatus social sobre las personas y recursos. 2) Logro; éxito personal mediante la demostración de competencia según criterios sociales. 3) Hedonismo; placer y gratificación sen- sual para uno mismo. 4) Estimulación; entusiasmo, novedad y reto en la vida. 5) Autodirección; pensamiento independiente y elección de la acción, creatividad, exploración. 6) Universalismo; comprensión, aprecio, tolerancia y protección del bienestar de todas las personas y de la naturaleza. 7) Benevolencia; preservación e intensificación del bienestar de todas las personas con las que uno está en contacto personal frecuente. 8) Tradición; respeto, compromiso y aceptación de las costumbres e ideas que proporcionan la cultura tradicional o la religión. 9) Conformidad; restricción de las acciones, inclinaciones e impulsos que pudiesen molestar o herir a otros y violar expectativas o normas sociales. 10) Seguridad; seguridad, armonia y estabilidad en la sociedad, de las relaciones y de sí mismo.

El mismo autor establece la existencia de una serie de relaciones dinámicas entre estos valores, considerando que algunos de ellos son compatibles entre sí mismos, mientras que otros son incompatibles. Esta relación entre los valores conforma una estructura coherente y ordenada que permite la localización de las distintas relaciones de compatibilidad, entre valores cercanos, y conflicto, entre los valores opuestos (Ver Fig.1).

Desde este enfoque teórico, Pons y Berjano (1999) realizaron un estudio sobre los factores relacionados con el consumo de alcohol en 1.100 jóvenes del municipio de Valencia. Para ello aplicaron una serie de cuestionarios de entre los que se destaca el VAL-89 que, mediante un conjunto de 56 items, evalúa las preferencias valorativas de los jóvenes. Así, estos autores encontraron que el consumo abusivo de alcohol está mediado por el mantenimiento de valores como la búsqueda de placer y estimulación (hedonismo y estimulación) y alejado del deseo expreso de bienestar y felici- 
dad tanto para el endogrupo como para el exogrupo (benevolencia y universalismo), y de la aceptación de tradiciones y convenciones sociales (tradición y conformidad).

En resumen, los valores se muestran como principios que guiando el comportamiento de los individuos, poseen una poderosa capacidad explicativa y de intervención a nivel psicosocial. Además existen investigaciones previas que han señalado con éxito la asociación existente entre el uso de drogas y los valores (Megias, 2000; Pons y Berjano, 1999; Rojo, Valdemoro y Cervera, 1994; Saiz, 2007), y por otra parte, distintos investigadores han propuesto su utilización en diversas áreas de la salud (Schwartz e Invar-Saban, 1988; Martínez-Sánchez y Ros, 2001).

\section{d) Rasgos de Personalidad y Drogas}

Desde una perspectiva psicosocial ${ }^{2}$, se entiende por rasgo de personalidad (Young, 1969, p.42) "un aspecto muy generalizado de la organización de la personalidad, que es relativamente independiente de cualquier objeto particular. (...) un rasgo es algo semejante a una actitud general, pero los rasgos carecen de la dirección de las actitudes".

Numerosos estudiosos (Digman, 1990; Goldberg, 1990; McCrae y John, 1992) han debatido, probado y consensuado la posibilidad de reducir y concretar el número de rasgos de personalidad a únicamente cinco rasgos o factores. Así, Digman (1990) describe los "Cinco Factores" de la siguiente manera: 1) Extraversión; también conocido como Energía, representa la polaridad propuesta por
Eysenck de Extroversión-Introversión. 2) Afabilidad; la cual consiste en la dimensión que envuelve más aspectos relacionados con la humanidad, como el altruismo, el cuidado emocional o los celos y la hostilidad. 3) Tesón; es considerado como la voluntad de lograr algo. 4) Estabilidad Emocional; el cual al enfrentarse con Neuroticismo, recuerda la segunda dicotomía propuesta por Eysenck, en donde se supone que individuos bajos en neuroticismo son tranquilos, fuertes, frios y emocionalmente controlados. Y 5) Apertura Mental (o Intelecto); indicando que altas puntuaciones en este factor revelan una personalidad culta, informada, interesada por las nuevas cosas y diferentes culturas.

Berrocal, Ortiz-Tallo, Fierro y Jiménez (2001) midieron rasgos de personalidad por medio del cuestionario BFQ (Caprara, Barbaranelli y Borgogni, 2001) y trastornos clínicos de personalidad por medio de otras escalas como el "Clinical Assessment Questionnaire", en una muestra de 47 sujetos adictos a la heroína incorporados en un programa de rehabilitación de "Proyecto Hombre". Para su estudio compararon las puntuaciones de este grupo de adictos con otro grupo control, así como con el grupo normativo que ofrecen los mismos cuestionarios. A pesar de encontrar mayores diferencias en las puntuaciones clinicas, los autores hallaron que el grupo de adictos puntuaba más bajo que los grupos de contraste en: tesón, apertura mental y estabilidad emocional (éste sólo significativo en las mujeres).

Pedrero $(2002,2003)$ también realizó diversas investigaciones sobre la personalidad del consumidor de drogas. En su estudio de 2002, su primer objetivo fue

\footnotetext{
${ }^{2}$ A pesar de que los rasgos de personalidad son elementos conceptuales típicamente recurridos desde la Psicología Clínica, existe una importante tradición que apoya la incorporación práctica y teórica de éstos, también, dentro de la Psicología Social (Young, 1969, Snyder y Cantor, 1998).
} 
el de analizar la estructura de la personalidad de los usuarios de un Centro de Atención a Drogodependencias (CAD). Y para ello aplicó el BFQ a 158 sujetos demandantes de tratamiento por abuso 0 dependencia de alcohol, cocaina o heroína. De esta forma, el autor encuentra que, específicamente en los consumidores de cocaina, sus puntuaciones más bajas se concentran en la escala de "estabilidad emocional", y sus dos subescalas, "control de las emociones" y "control de impulsos". En una investigación posterior, Pedrero (2003) intenta comparar las puntuaciones básicas de personalidad obtenidas por dos técnicas distintas (BFQ y MCMI-II). Tal y como si fuese la réplica del anterior estudio, la aplicación del BFG volvió a informar de bajas puntuaciones en "estabilidad emocional" para casi todos los tipos de consumidores. Las subescalas de "control de emociones" y "control de impulsos" también repitieron puntuaciones bajas.

En resumen, los rasgos de personalidad, siendo características que los individuos muestran con cierta consistencia en distintas situaciones, poseen una capacidad descriptiva así como de intervención muy importante para el profesional en drogodependencias. En este epígrafe se ha señalado la relevancia del Modelo de los Cinco Factores, así como los hallazgos empíricos que relacionan exitosamente el uso de drogas con determinados factores, especialmente estabilidad emocional y tesón.

\section{e) Relación entre Valores y Rasgos de Personalidad}

Antes de proponer un modelo que integre los anteriormente descritos, será necesario resaltar las diferencias encontradas entre rasgos de personalidad y valores personales. Según Bilsky y Schwartz (1994, p. 165): “1) los rasgos de personalidad son típicamente vistos como patrones de conducta observables, mientras que los valores son criterios que los individuos usan para juzgar la deseabilidad de la conducta, la gente y eventos. 2) Los rasgos de personalidad varian en términos de cuanta característica de personalidad un individuo exhibe (y son independientes de cualquier objeto particular), mientras que los valores varían en términos de la importancia que los individuos atribuyen a objetivos particulares. 3) Los rasgos de personalidad describen acciones que presumiblemente surgen de "la forma en como la gente es" sin importar sus intenciones, mientras que los valores se refieren a los objetivos intencionales de los individuos que están a disposición de la conciencia”.

En sintesis, mientras que los rasgos de personalidad son atribuciones que se realizan a los sujetos desde afuera según su comportamiento observable, los valores son experimentados como demandas o necesidades que uno mismo adquiere por convicción intencional. La necesidad de aplicar ambos en combinación en el área de las drogodependencias, resulta más evidente aun, cuando se toma en cuenta las especificidades de su acción en la explicación conductual: mientras que los rasgos de personalidad se refieren a aspectos relativamente estables y de menor control cognitivo, más automáticos si cabe, los valores se aplican a aspectos del comportamiento humano también relativamente estables, pero con un mayor control volitivo, que implica evaluación de alternativas y acción reflexiva.

Roccas, Sagiv, Schwartz y Knafo (2002) relacionaron y compararon los "Cinco Grandes Factores" con las prioridades valorativas que Schwartz (2001) propone. Para llevar a acabo este estudio los autores aplicaron el Cuestionario de Valores de Schwartz (2001) y el Inventa- 
rio de Personalidad NEO-PI (Costa y McCrae, 1999) a 246 estudiantes israelíes. Además, con el fin de conocer el alcance explicativo de estas dos variables, las relacionaron con otras dos: Religiosidad y Afecto positivo. Esencialmente, tal y como se observa en la Figura 1, encontraron importantes correlaciones entre todos los rasgos y valores (ejem. extroversión y estimulación; apertura a la experiencia y autodirección; afabilidad y benevolencia; tesón y seguridad), con excepción del rasgo estabilidad emocional que mostró una asociación muy pequeña con los valores.

En cuanto a la asociación de valores y rasgos con la religiosidad, puede decirse que los valores correlacionaron en mayor medida que los rasgos, explicando un $42 \%$ de la varianza frente al $8 \%$ que explicaron estos últimos. Mientras que los rasgos de personalidad tuvieron una mayor participación en el porcentaje de explicación de la varianza del afecto positivo ( $11 \%$ vs. 5\%). Esta forma de comportarse con terceras variables (religiosidad y afectividad), llevaron a los autores a concluir que (Roccas et al., 2002, p.798): "Los valores podrían influir más fuertemente actitudes y conductas que están bajo control cognitivo, control volitivo, mientras que los rasgos podria afectar más fuertemente tendencias y conductas sujetas a poco control cognitivo". Estos datos fueron posteriormente repetidos por Olver y Mooradian (2003) (Ver Fig. 1).

\section{Psicologia Social de la Salud sobre el consumo adictivo de Cocaina: Un modelo psicosocial sintético}

Hasta aquí se han descrito algunas variables que, desde la Psicología Social, se han aplicado con mayor éxito y mejores resultados al campo de la salud y específicamente al consumo de drogas.
Falta por lo tanto ofrecer un modelo que, a modo de resumen, logre reunir los avances que esta ciencia posee y facilite al investigador y a los distintos profesionales sus labores dentro del campo de la prevención y terapéutica de la adicción a la cocaína. El Modelo Psicosocial Sintético para el Consumo Adictivo de Cocaína se podría resumir de la siguiente manera (Ver Fig. 2).

En primer lugar, podría decirse que, además de la determinación biológica de la adicción, el consumo adictivo de cocaína en el sujeto está influido por dos grandes bloques: el bloque social y el bloque psicológico. Ambos aspectos de influencia son totalmente interdependientes, resultando aun complicado establecer el grado en que cada uno determina al otro. En la combinación y articulación de ambos deberíamos poder encontrar la respuesta para la adicción particular de cada sujeto. El bloque psicológico estaría constituido por factores como: Valores, Rasgos de personalidad, Actitudes, Control Conductual Percibido, Intención, Autoestima y Estrategias de afrontamiento. Mientras que el bloque social lo formarian los factores: Norma subjetiva, Posición social, Sucesos estresantes, Alineación, Apoyo social y Caracteristicas ambientales.

Según este modelo, no existe un perfil único del consumidor-adicto de cocaina, sino que los diferentes factores se combinan de forma especial en cada sujeto actuando a su vez como factores de riesgo y/o protección. Sin embargo, sería posible realizar algunas puntualizaciones: tomando en cuenta la TCP (Ajzen, 1991), cuando existen unas actitudes favorables hacia el consumo de cocaína, la creencia de que el grupo de referencia apoya este consumo (norma subjetiva) y la creencia de que se es capaz de controlar el consumo de cocaina (o de que es totalmente incapaz de dejar de consumir), el sujeto 
Figura 1. Modelo teórico de las relaciones entre los tipos motivacionales (Schwartz, 2001) y los rasgos de personalidad según el Modelo de los Cinco Factores (Roccas et al., 2002)

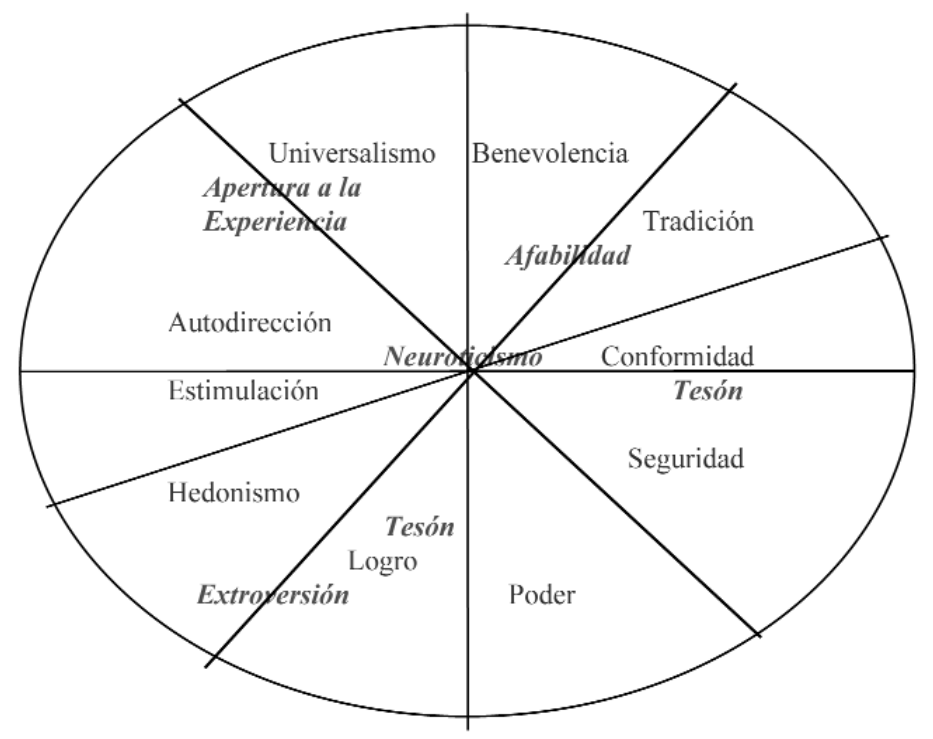

Nota: La localización de los rasgos de personalidad dentro de la estructura circular de Schwartz (2001) se ha realizado tomando en cuenta las correlaciones que encontraron Roccas et al. (2002) no basándose en un SSA, por lo tanto, el resultado gráfico es aproximado, no exacto, y sólo se expone con el fin de ejemplificar las relaciones de compatibilidad e incompatibilidad entre valores y rasgos de personalidad.

tiene mayores probabilidades de seguir consumiendo la droga. Además, si el individuo valora el hedonismo y la estimulación y posee como rasgo de personalidad predominante la extroversión, coincidirá con un patrón de consumo de la droga con fines recreativos (Calafat, et al., 2001). Por otra parte, cuando el sujeto no conserva una clara jerarquía de valores y su rasgo de personalidad predominante es el neuroticismo o la baja estabilidad emocional, el consumo de cocaina puede estar asociado con motivos no recreativos o festivos, y la situación en la que se haya requiere de una reorganización axiológica, tanto para fomentar la rehabilitación como en programas de prevención. Asimismo, tomando en consideración el Modelo Psicosociológico para el Estudio de la Salud Mental (Sánchez, Garrido y Álvaro, 2003), la Autoestima y las Estrategias de afrontamiento serán dos factores sumamente importantes en la mediación entre la posición social del sujeto y su conducta y situación personal. De este modelo se desprende también que, una posición social desfavorable puede colocar al sujeto en unas condiciones de vida que creen estrés y alineación, lo cual podría desencadenar en un tipo de dependencia de la cocaína más marginal, y no exclusivamente recreativa. 
Figura 2. Modelo Psicosocial Sintético para el Consumo Adictivo de Cocaina

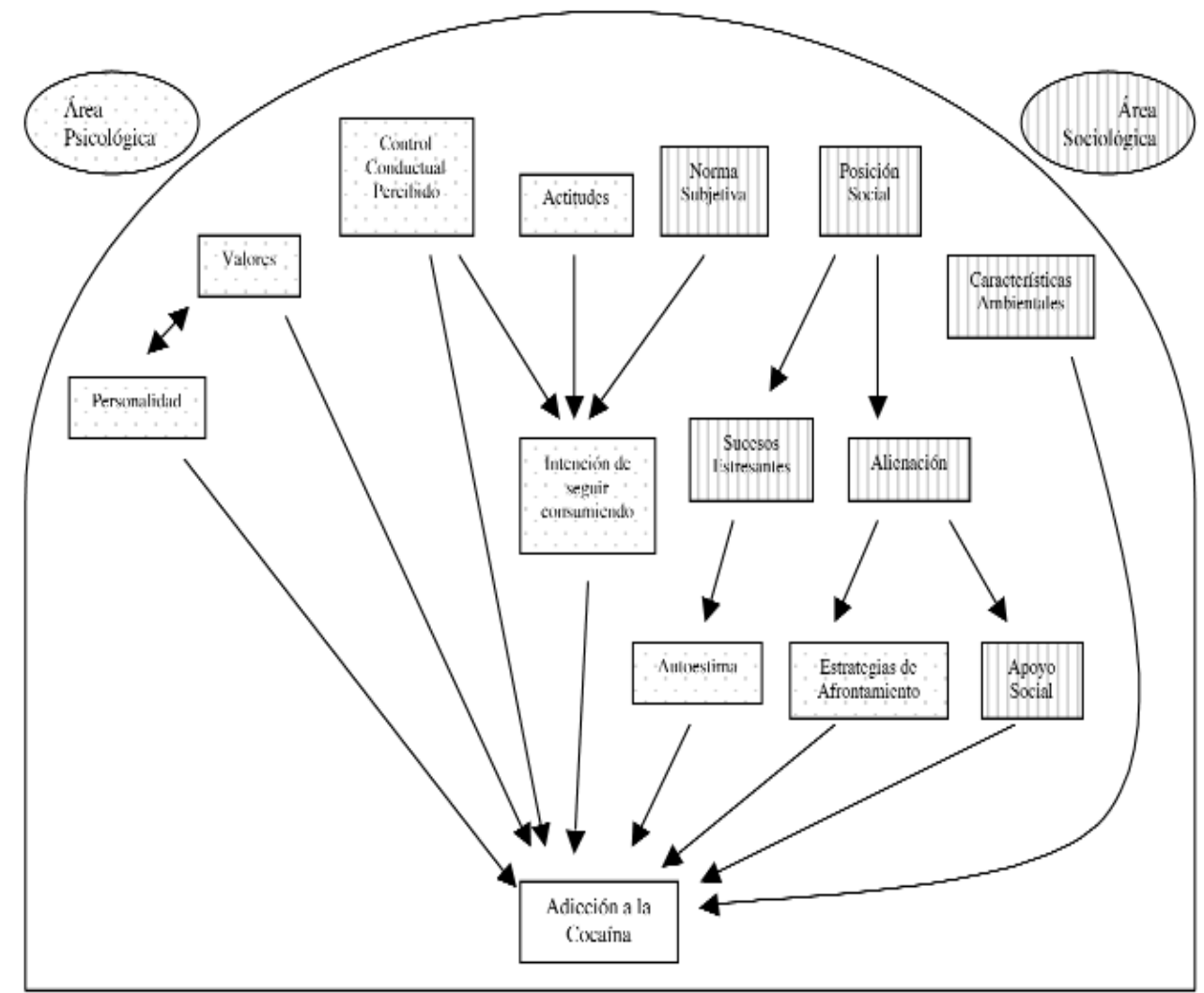

De esta manera, como ya lo han hecho notar otros autores (Moral, Ovejero, Sirvent y Rodríguez, 2005), se establece que en la prevención será insuficiente la modificación de las actitudes con la mera exposición informativa, si el contexto sociocultural en el que se mueven los potenciales consumidores motiva el uso de cocaína, y tanto la norma subjetiva como el apoyo social y el propio clima socioeconómico invitan al consumo. Se recomienda por lo mismo, desde este Modelo Psicosocial, el trabajo preventivo en dos frentes: el individual, favoreciendo valores, actitudes, explorando el control conductual percibido y estrategias de afrontamiento incompatibles con el consumo de cocaína; y el social, devaluando la imagen social (norma subjetiva) del consumo de cocaína y apoyando la mejor posición social y condiciones de vida de los individuos.

En cuanto a la aplicación de este modelo en el trabajo terapéutico, se debe enfatizar la importancia que valores, rasgos de personalidad, autoestima y estrategias de afrontamiento poseen para el individuo que necesita re-adaptarse a la sociedad. Por una parte, los valores y rasgos de personalidad se complementan para evaluar y estructurar las prioridades del sujeto y su situación personal, mientras que la importancia de la autoestima reside en la especial capacidad que ésta posee como mediadora entre la 
situación personal del sujeto, su posición social y la influencia del estigma social (Saiz, 2008a). Por último, mientras que el terapeuta no pueda modificar las características ambientales y la realidad de la estructura socioeconómica del sujeto, será necesario un trabajo intensivo con los recursos-estrategias que permitan al individuo "enfrentarse" a un medio que continuará, probablemente, ofreciendo al sujeto los mismos obstáculos y "tentaciones" que le llevaron al consumo adictivo (Alexander, 2001, 2006; Valverde, 1996).

\section{Conclusiones y Discusión}

Actualmente el consumo de drogas ha experimentado un importante cambio cuantitativo y cualitativo. Mientras que el uso de heroina se está reduciendo lentamente, otras drogas han elevado su consumo asociándose a una representación social de diversión y coincidiendo con un clima social recreativo y consumista (Calafat et al., 2000, Comas, 2001). De hecho, el lugar de peligrosidad que en los años 80 poseía la heroína ahora es ocupado por la cocaina (Megias, 2004), sin que por ello se haya logrado aun reducir su consumo y evitar la continua serie de problemas personales y sociales que están ocurriendo en aumento.

En el tema de las adicciones a sustancias, el análisis que se procura llevar siempre a cabo es el análisis bio-psicosocial. Este compuesto de tres términos implica que el abordaje del problema desde el ámbito médico con exclusividad es insuficiente, mientras que el análisis psicológico de la adicción, aunque puede conducir a resultados plausibles, deja sin tocar el contexto en el cual ha surgido la dependencia y el ambiente en el que el sujeto habrá de re-insertarse. De esta manera, la aproximación "biopsicosocial" a la salud (Pitts, 1998, p.9) "reconoce las bases biológicas y genéticas de muchas enfermedades, incluye el rol de elementos psicológicos como creencias, comportamientos y cogniciones en el desarrollo de las enfermedades y reconoce que los contextos sociales, económicos y culturales tendrán gran impacto en la salud”.

Por esto, con el presente artículo se ha pretendido rescatar aquellas herramientas que la Psicología Social de la Salud y la Salud Mental poseen para el estudio y trabajo en este área y, tras revisar algunos modelos teóricos e investigaciones, se ha ofrecido un modelo teórico que resuma las mismas y posibilite una posición desde la cual analizar e intervenir sobre el problema.

No obstante, a pesar de los avances en la comprobación empírica de este modelo (Saiz, 2008b), resulta necesario subrayar el carácter tentativo del mismo, así como la necesidad de realizar estudios cualitativos y cuantitativos, que permitan obtener inferencias basadas en la evidencia científica.

En conclusión, la drogadicción es un fenómeno multifactorial, el cual requiere un trabajo en equipo multidisciplinar, una perspectiva integral y que considere el contexto social y cultural en el cual surge el problema. 


\section{BIBLIOGRAFÍA}

Alexander, B. K. (2001). The roots of addiction in free market society. Recuperado el 10 de junio de 2006 de http://www.policyalternatives.ca.

Alexander, B. K. (2006). Beyond Vancouver's "Four Pillars". International Journal of Drug Policy, $17,118-123$.

Ajzen, I. (1991). The Theory of Planned Behavior. Organizational Behavior \& Human Decision Processes, 50, 179-211.

Ajzen, I. y Fishbein, M. (1980). Understanding attitudes and predicting social behavior. EEUU.: Prentice Hall

Álvaro, J. L. y Páez, D. (1999). Psicología social de la salud mental. En J. L. Álvaro, A. Garrido y J. R. Torregrosa (Eds.). Psicología social aplicada. España: McGrawHill

Becoña, E. (1999). Bases Teóricas que sustentan los programas de prevención de drogas. España: PNSD.

Becoña, E. (2002). Bases científicas de la prevención de las drogodependencias. España: PNSD.

Berrocal, C., Ortiz-Tallo, M., Fierro, A. y Jiménez, J. A. (2001). Variables clinicas y de personalidad en adictos a heroína. Anuario de Psicología, $32,67-87$.

Bilsky, W. y Schwartz, S. H. (1994). Values and personality. European Journal of Personality, 8, 163-181.

Calafat, A., Juan, M., Becoña, E., Fernández, C., Gil, E., Palmer, A., et al. (2000). Salir de marcha y consumo de drogas. Madrid: PND - Caja Madrid.

Calafat, A., Juan, M., Becoña, E., Fernández, C., Gil, E. y Llopis, J. J. (2001). Vida social de la cocaina. Adicciones, 13, (Supl, 2), 61-104.

Caprara, G. V., Barbaranelli, C. y Borgogni, L. (2001). Cuestionario "Big Five". Madrid: TEA.

Comas, D. (2001). La representación social del fin de semana de los jóvenes. Revista de Estudios de Juventud, 54, 71-78.

Costa, P. I. y McCrae, R. R. (1999). NEO PI-R. Madrid: TEA.
Digman, J. M. (1990). Personality structure: emerge of the five-factor model. Annual Review of Psychology, 41, 417-440.

Goldberg, L. R. (1990). An alternative "Description of Personality"; the Big-Five Factor structure. Journal of Personality and Social Psychology, 59, 1216-1229.

Hulten, R., Bakker, A. Lodder, A., Teeuw, K., Bakker, A. y Leufkens, H. (2003). The impact of attitudes and beliefs on lengh of benzodiazepine use: a study among inexperienced and experienced benzodiazepine users. Social Science and Medicine, $56,1345-1354$.

Humphrey, R., O`Malley, P., Johnston, L. y Bachman, J. (1988). Bases of power, facilitation effects, and attitudes and behavior: direct, indirect, and interactive determinants of drug use Social Psychology Quarterly, 51, 329-345.

Levy, S. y Pierce, P. (1989). Predicting intention to use cocaine in teenagers in Sydney, Australia. Addictive Behaviors, 14, 105-111.

Martínez-Sánchez, E. y Ros, M. (2001). Los significados de la salud y la salud como valor. En M. Ros y V. V. Gouveia (Coords.). Psicología social de los valores humanos. España: Biblioteca Nueva.

McCrae, R. y John, O. (1992). An Introduction to the Five-Factor Model and Its Applications. Journal of Personality, 60, 175-215.

Megías, E. (Dir.) (2000). Los valores de la sociedad española y su relación con las drogas. España: Fundación la Caixa.

Megias, E. (Dir.). (2004). La percepción social de los problemas de drogas en España. España: FAD.

Moral, M., Ovejero, A., Sirvent, C. y Rodríguez, F., (2005). Prevención e intervención psicosocial sobre el consumo juvenil de sustancias psicoactivas: Comparación de la eficacia preventiva de cuatro programas. Intervención Psicosocial, 14, 198-208.

OED. (2005). Informe 2004. Madrid: Ministerio del Interior.

Olver, J. y Mooradian, T. (2003). Personality traits and personal values: a conceptual and empirical integration. Personality and Individual Differences, 35, 109-125. 
Parrott, A. (1998). Social Drugs: effects upon health. En M. Pitts y K. Phillips (Eds.): The psychol ogy of health. London: Routledge.

Pedrero, J. (2002). Evaluación de la personalidad de sujetos drogodependientes que solicitan tratamiento mediante el Big-Five Questionnaire. Trastornos Adictivos, 4, 138-150.

Pedrero, J. (2003). Los trastornos de la personalidad en drogodependientes desde la perspectiva de los cinco grandes factores. Adicciones, 15, 203220

Pitts, M. (1998). An introduction to health psychology. En M. Pitts y K. Phillips (Eds.). The psychology of health. London: Routledge.

Plan Nacional sobre Drogas. (2004). Encuesta Estatal sobre el uso de drogas en enseñanza secundaria. Disponible en: www.msc.es/pnd.

Pons, J. y Berjano, E. (1999). El consumo abusivo de alcohol en la adolescencia: un modelo explicativo desde la psicología social. España: PND.

Roccas, S., Sagiv, L., Schwartz, S. H. y Knafo, A. (2002). The Big Five Personality Factors and Personal Values. Personality and Social Psychology Bulletin, 28, 789-801.

Rodríguez, J. y García, J. A. (1999). Psicología social de la salud. En J. L. Álvaro, A. Garrido y J. R. Torregrosa (Eds.). Psicología social aplicada. España: McGrawHill.

Rojo, J., Valdemoro, C. y Cervera, G. (1994). Análisis de los valores en toxicómanos, medidos mediante la escala de valores de Rokeach. Anales de Psiquiatría, 10, 272-278.

Ros, M. (2001). Valores, actitudes y comportamiento: una nueva visita a un tema clásico. En $\mathrm{M}$ Ros y V. V. Gouveia (Eds.). Psicología social de los valores humanos. España: Biblioteca Nueva.

Rutter, D. R., Quine, L. y Chesham, D. J. (1993). Social psychological approaches to health London: Harvester-Wheatsheaf.

Saiz, J. (2007). El abuso de cocaina, ¿Problema de oferta o de demanda social?: un estudio transcultural y correlacional que compara variables macrosociales, económicas y culturales. Adicciones, 19, 35-44.

Saiz, J. (2008a). El estigma de la cocaína. Revista Española de Sociología, 10.

Saiz, J. (2008b). Un estudio del consumo de cocaina en la Comunidad de Madrid desde la Psicologia Social. Athenea Digital, 13, 325-337.

Sánchez, E., Garrido, A. y Álvaro, J. L. (2003). Un modelo psicosociológico para el estudio de la salud mental. Revista de Psicología Social, 18, 17-33.

Schifter, D. y Ajzen. I. (1985). Intention, Perceived Control, and Weight Loss: An Application of the Theory of Planned Behavior. Journal of Personality and Social Behavior, 49, 843-851.

Schwartz, S. y Bilsky, W. (1987). Toward a Universal Psychological Structure of Human Values. Journal of Personality and Social Psychology, 53, 550-562.

Schwartz, S. H. e Invar-Saban, N. (1988). Value self-confrontation as a method to aid to weight loss. Journal of Personality and Social Psychology, $54,396-404$.

Schwartz, S. H. (2001). ¿Existen aspectos universales en la estructura y contenido de los valores humanos?. En M. Ros y V. V. Gouveia (Eds.). Psicología social de los valores humanos. España: Biblioteca Nueva.

Snyder, M. y Cantor, N. (1998). Understanding personality and social behavior: a functionalist strategy. En D. T. Gilbert, S. T. Fiske y G. Lindzey. The Handbook of Social Psychology. USA.: MsGrawHill.

Stroebe, W. y Stroebe, M. (1995). Social Psychol ogy and Health. Reino Unido: Open University Press.

UNODC. (2007). World Drug Report 2007. Recuperado el 27 de junio de 2007 de: www.unodc.org.

Valverde, J. (1996). Vivir con la droga. Madrid: Pirámide.

Young, K. (1969). Psicología Social de la Personalidad. Buenos Aires: Paidós. 